\title{
Top pair cross section measurements and event modelling with the CMS detector
}

\section{James Keaveney on behalf of the CMS collaboration}

DESY, Notketraße 85, 22607 Hamburg, Germany

E-mail: james.keaveney@desy.de

\begin{abstract}
Recent measurements of inclusive and differential top quark pair production cross sections in proton collisions at centre of mass energies of 5.02, 7, 8 and $13 \mathrm{TeV}$ from the CMS experiment are presented. With the exception of the top quark transverse momentum distribution where a softer spectrum is observed in data with respect to predictions, all result are consistent with the expectations of the standard model. Results on measurements of the jet and b-jet activity in top quark pair events and a search for the production of four top quark events are also presented.
\end{abstract}

38th International Conference on High Energy Physics

3-10 August 2016

Chicago, USA 


\section{Introduction}

The enormous datasets provided by the LHC contain copious amounts of top quark pair $(t \bar{t})$ events allowing precise measurements of the inclusive and differential $t \bar{t}$ cross sections. As the higher order corrections to $t \bar{t}$ production are large, these measurements test the predictive power of perturbative QCD calculations. Similarly, $t \bar{t}$ observables at particle level can constrain the parton shower and hadronisation modelling. Due to the large mass of the top quark, $t \bar{t}$ cross sections are sensitive to the parton distribution function (PDF) of the gluon. Hence these measurements have the potential to reduce the PDF uncertainties. The experimental signature of a $t \bar{t}$ event is is similar to that of numerous beyond the standard model (BSM) scenarios, e.g, the production of a $Z^{\prime}$ particle decaying to $t \bar{t}$ or the production of a pair of stop squarks with decays to $t \bar{t}$ and additional SUSY particles. In scenarios where the signature is practically indistinguishable from standard model (SM) production, the new physics may be manifest as modifications in the measured rates and kinematics $t \bar{t}$ production. Thus these measurements can increase the sensitivity of searches for BSM physics by seeking signs of BSM physics in deviations of $t \bar{t}$ measurements from SM expectations. In addition, inclusive and differential measurements of $t \bar{t}$ with additional jets, $(t \bar{t}+$ jets), bottom quarks $(t \bar{t} b \bar{b})$ or top quarks $(t \bar{t} t \bar{t})$ production are presented.

The CMS collaboration [1] has published many such measurements in a range of final states and in both boosted and resolved regimes at centre of mass energies of 5.02, 7, 8 and $13 \mathrm{TeV}$. In this note, only a small number of recent results are described.

\section{Inclusive $t \bar{t}$ cross section in the dilepton channel at $5.02 \mathrm{TeV}$}

A measurement of the inclusive $t \bar{t}$ cross section in the dilepton channel using $26 \mathrm{pb}^{-1}$ of proton collision data with a centre of mass energy of $5.02 \mathrm{TeV}$ is presented [2]. The low centre of mass energy of this dataset corresponds to higher Bjorken-x values for $t \bar{t}$ production. As PDF uncertainties for the gluon are large at high Bjorken-x, this measurement has the potential to reduce PDF uncertainties. Moreover, a future measurement of $t \bar{t}$ production in nuclear collisions at 5.02 $\mathrm{TeV}$ will benefit from having this measurement as a reference. At trigger level, events required to contain one muon with a transverse momentum $\mathrm{P}_{T}>15 \mathrm{GeV}$. Offline, events are required to contain one muon and one electron candidate of opposite charge satisfying the requirements $\mathrm{P}_{T}>18 \mathrm{GeV}$, $|\eta|<2.1$ and $>20 \mathrm{GeV},|\eta|<2.4$ respectively. Events with a dilepton invariant mass less than 20 $\mathrm{GeV}$ are removed in order to suppress background events from low-mass Drell-Yan production and the decay of heavy resonances. The residual backgrounds are mainly estimated from data with the exception of single top quark and $\mathrm{W}$ boson events (tW) and $\mathrm{W}$ boson plus an additional electroweak boson events (WV) which are estimated using simulation. In 1, distributions for the multiplicity of jets and invariant mass of the dilepton system in data and simulation are shown. Good agreement between data and simulation is observed with a high purity of $t \bar{t}$ events in the selected data.

The cross section is calculated by subtracting the estimated total number of background events from the number of events in data and then correcting for detector efficiencies and acceptance. The final result is:

$$
\sigma(p p \rightarrow t \bar{t})=82 \pm 20(\text { stat. }) \pm(\text { syst. }) \pm(\text { lumi. })=82 \pm 23(\text { total }) p b
$$



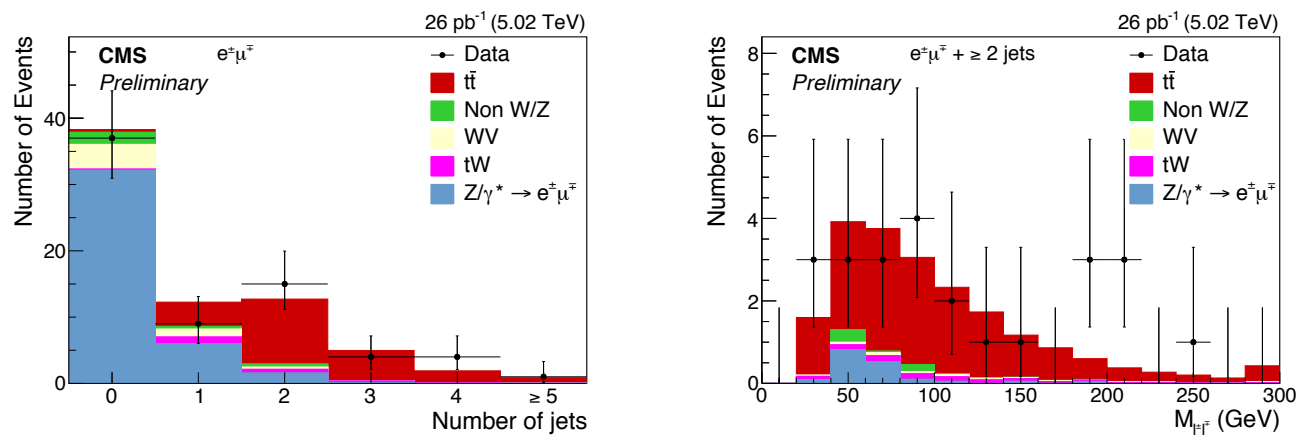

Figure 1: The distributions for the multiplicity of jets (left) and invariant mass of the dilepton system (right) in data and simulation are shown.

which is in good agreement with the NNLO QCD prediction [3].

\section{Inclusive $t \bar{t}$ cross section in the lepton + jets channel at $13 \mathrm{TeV}$}

A measurement of the inclusive $t \bar{t}$ cross section in the lepton + jets channel using $2.3 \mathrm{fb}^{-1}$ of $13 \mathrm{TeV}$ proton collision data is presented [4]. The data are selected using triggers that require an isolated muon or electron with minimum $\mathrm{P}_{T}$ of 20 or $22 \mathrm{GeV}$ respectively. Offline, events are required to contain a an isolated lepton candidate passing lepton identification requirements with $\mathrm{P}_{T}>30 \mathrm{GeV},|\eta|<2.1$. Selected events are categorised according to the number of jets, b-jets, lepton flavour and lepton charge, resulting in 44 exclusive categories. Within each category, the invariant mass of the $b$-jet and lepton system is used as a discriminating variable to further separate signal from background. The $t \bar{t}$ is extracted via a simultaneous fit of signal and background in all categories. Systematic uncertainties are accommodated in the fit via nuisance parameters. The final result is

$$
\sigma(p p \rightarrow t \bar{t})=834.6 \pm 2.5(\text { stat. }) \pm 22.8(\text { syst. }) \pm 22.5(\text { lumi. }) p b
$$

which is in good agreement with the latest prediction at NNLO+NNLL accuracy in QCD.

\section{Differential $t \bar{t}$ cross section at particle level in the dilepton channel at $13 \mathrm{TeV}$}

A measurement of the normalised differential $t \bar{t}$ cross section in the dilepton channel are presented [5]. The measurements utilise $2.2 \mathrm{fb}^{-1}$ of proton collision data with a centre of mass energy of $13 \mathrm{TeV}$. At trigger level, events are required to contain two leptons (electrons or muons). Offline, events are required contain two isolated, opposite-charge, leptons satisfying loose kinematic requirements and two reconstructed jets, one of which must be identified as originating from a b-quark. In the same flavour channels (events containing two electrons or two muons), events are required to have dilepton invariant mass far from the $\mathrm{Z}$ mass and a missing transverse momentum of at least $40 \mathrm{GeV}$. The kinematics of the top quarks in the events are estimated using an analytical reconstruction method. The differential cross section is measured in a fiducial phase space at particle level defined with kinematic requirements looser than those of the offline selection. After 
the subtraction of the estimated background contributions and corrections for detector efficiencies, events are unfolded to this fiducial phase space. With the exception of the top quark $\mathrm{P}_{T}$ spectrum which is found to be softer in data than in simulation, all measured distributions agree with the SM expectation. In figure 3 the differential cross section is shown as a function of the top quark $\mathrm{P}_{T}$ and the invariant mass of the $t \bar{t}$ system.
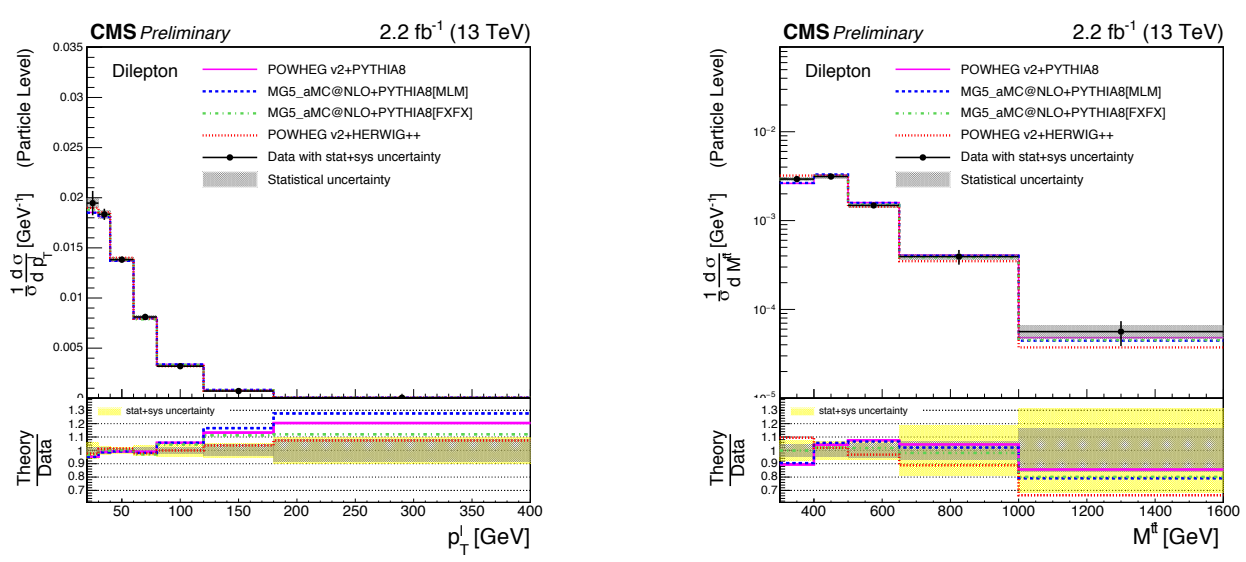

Figure 2: The differential cross sections in data and simulation are shown as a function of the top quark $\mathrm{P}_{T}$ (left) and the invariant mass of the $t \bar{t}$ system (right).

\section{Double differential cross section measurement in the dilepton channel at $8 \mathrm{TeV}$.}

A measurement of the double differential cross section measurement in the dilepton channel using $19.7 \mathrm{fb}^{-1}$ of proton collision data at $8 \mathrm{TeV}$ is presented [6]. At trigger level, events are required to contain an electron and a muon, one of which is required to have $\mathrm{P}_{T}>17 \mathrm{GeV}$ and the other $\mathrm{P}_{T}>8 \mathrm{GeV}$. Offline, the events are required to contain one isolated electron and one isolated muon, each satisfying the requirements $\mathrm{P}_{T}>20 \mathrm{GeV},|\eta|<2.4$ with the invariant mass of the dilepton system required to be greater than $20 \mathrm{GeV}$. Furthermore, events are required to contain two jets with $\mathrm{P}_{T}>8 \mathrm{GeV}$, one of which must be identified as originating from a b-quark. A kinematic reconstruction technique is applied in order to deduce the kinematics of the top quarks. After the subtraction of backgrounds and corrections for detector effects, events are unfolded to the parton level. The cross section is evaluated in bins differential in two variables. Generally, there is good agreement between the data and the simulation. However, the simulation shows a harder top quark $\mathrm{P}_{T}$ spectrum than the data. The rapidity of the $t \bar{t}$ system is less central in simulation than in data, while the distribution of top quark rapidity $\mathrm{y}(\mathrm{t})$ is more central than the data. In figure 3 , the differential cross sections in data and simulation are shown as a function of the rapidity and invariant mass of the $t \bar{t}$ system and the $\mathrm{P}_{T}$ and rapidity of the top quarks. The sensitivity of these measurements to the parton distribution function of the gluon at high Bjorken $\mathrm{x}$ is demonstrated in [6].

\section{Measurements of $t \bar{t}+$ jets in the lepton + jets channel at $8 \mathrm{TeV}$}

A measurement of the differential $t \bar{t}$ cross section as a function of the jet multiplicity in the 

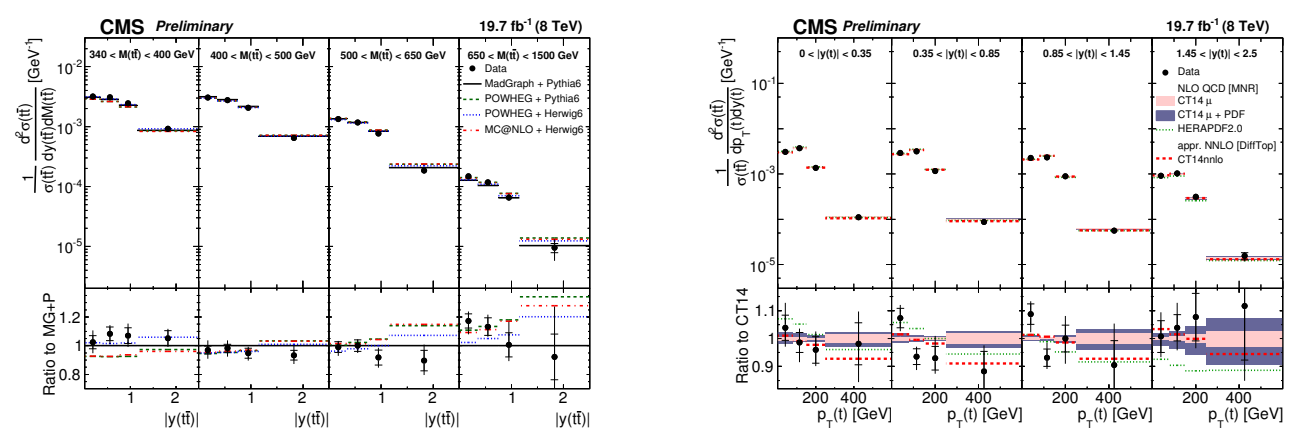

Figure 3: The differential cross sections in data and simulation are shown as a function of the rapidity and invariant mass of the $t \bar{t}$ system (left) and the $\mathrm{P}_{T}$ and rapidity of the top quarks (right).

lepton + jets channel are presented [7]. The measurement utilises $19.7 \mathrm{fb}^{-1}$ of $8 \mathrm{TeV}$ proton collision data. At trigger level, events are required to contain a muon with $\mathrm{P}_{T}>24 \mathrm{GeV},|\eta|<2.1$ or an electron with $\mathrm{P}_{T}>27 \mathrm{GeV},|\eta|<2.4$. Offline, the events are required to contain one electron or one muon, and at least four jets, two of which must be identified as originating from a b-quark. After subtraction of background components, the data are unfolded to the particle level. Two set of measurements are presented: one in a fiducial phase space and one in full phase space after a further unfolding has been applied. Overall, reasonable agreement is observed between data and the predictions from a range of Monte Carlo generators. All considered simulations predict too few events at very high jet multiplicities. The best agreement is observed for a simulation based on POWHEG for the matrix element and HERWIG for the parton shower.

\section{Measurements of $t \bar{t} b \bar{b}$ in the dilepton channel at $13 \mathrm{TeV}$}

A measurement of the cross sections of $t \bar{t}$ production in association with a pair of b-jets $(t \bar{t} b \bar{b})$ a pair of jets of any flavour $(t \bar{t} j j)$ and their ratio using dilepton final states with $2.32 \mathrm{fb}^{-1}$ of 13 $\mathrm{TeV}$ proton collision data is presented [8]. Events are recorded using dilepton triggers requiring an electron and a muon with a $\mathrm{P}_{T}$ threshold of $17 \mathrm{GeV}$. Offline, the events are required to contain one isolated electron and one isolated muon, each satisfying the requirements $\mathrm{P}_{T}>20 \mathrm{GeV},|\eta|<$ 2.4. Events are also required to contain four or more jets with $\mathrm{P}_{T}>30 \mathrm{GeV}$ and $|\eta|<2.4$, two of which must be identified as originating from a b-quark. After the event selection, the ratio of the number of $t \bar{t} b \bar{b}$ events to $t \bar{t} j j$ events is obtained from the data by fitting the b-tagging discriminator distributions. The cross section ratio is measured in a fiducial phase space at particle level by correcting for detector and selection efficiencies and then extrapolated to the full phase space by correcting for acceptance effects with the result

$$
\sigma(t \bar{t} b \bar{b}) / \sigma(t \bar{t} j j)=0.022 \pm 0.003(\text { stat. }) \pm 0.006(\text { syst. })
$$

The result is higher but compatible with the prediction from simulation based on the POWHEG generator for the Matrix Element and the PYTHIA generator for the parton shower. 


\section{Search for $t \bar{t} t \bar{t}$ in the lepton + jets and dilepton channels at $13 \mathrm{TeV}$}

A search for the SM production of events containing four top quarks is presented [9]. The search is performed in lepton plus jets and dilepton channels with $2.3 \mathrm{fb}^{-1}$ of proton collision data at $13 \mathrm{TeV}$. Offline selected events are divided into categories based on the multiplicities of jets and b-jets. A discriminator based on a BDT algorithm is used to further distinguish signal from backgrounds. The potential presence of an SM $t \bar{t} t \bar{t}$ signal in the data is investigated by fitting the predicted distributions for signal and background to data simultaneously in all categories while accounting for systematic uncertainties via nuisance parameters. As no evidence for the signal is observed, upper limits of $10.2 \times \sigma(t \bar{t} t \bar{t})^{S M}$ (observed) and $10.8_{-3.8}^{+6.7} \times \sigma(t \bar{t} t \bar{t})^{S M}$ (expected) are calculated.

\section{References}

[1] CMS Collaboration, The CMS experiment at the CERN LHC, JINST 3 S08004 (2008)

[2] CMS collaboration, First measurement of the top quark pair production cross section in proton-proton collisions at 5.02 TeV. CMS-PAS-TOP-16-015

[3] M. Czakon and A. Mitov, TOP++: a program for the calculation of the top-pair cross-section at hadron colliders, Comput. Phys. Commun. 185 (2014) 2930, doi:10.1016/j.cpc.2014.06.021, arXiv:1112.5675

[4] CMS collaboration, A measurement of the inclusive tt production cross section in proton-proton collisions at $13 \mathrm{TeV}$ using events with one isolated charged lepton and at least one jet. CMS-PAS-TOP-16-006

[5] CMS collaboration, Measurement of particle level differential tt cross sections in the dilepton channel at $13 \mathrm{TeV}$. CMS-PAS-TOP-16-007

[6] CMS collaboration, Measurement of double differential cross sections for top quark pair production in pp collisions at $8 \mathrm{TeV}$. CMS-PAS-TOP-14-013

[7] CMS collaboration, Measurement of the differential production cross section for top-quark pairs as a function of jet multiplicity in the lepton+jets final state at $8 \mathrm{TeV}$ with the CMS detector. CMS-PAS-TOP-15-006

[8] CMS collaboration, Measurement of the cross section ratio $\sigma(t \bar{t} b \bar{b}) / \sigma(t \bar{t} j j)$ using dilepton final states in pp collisions at $13 \mathrm{TeV}$ CMS-PAS-TOP-16-010

[9] CMS collaboration, Search for standard model production of four top quarks in proton-proton collisions at $13 \mathrm{TeV}$ CMS-PAS-TOP-16-016 